1 Context, competition, and symbiont-induced bet-hedging between Dictyostelium

2 discoideum and Paraburkholderia

3

4

9 Department of Biology, Washington University in St. Louis

10

11

$12 *$ Corresponding Author

13 E-mail: tjscott@wustl.edu

14

15

16

17

18

19

20

21 Keywords: symbiosis, context-dependence, competition, bet-hedging, $D$. discoideum,

\title{
22 Paraburkholderia
}

23 


\section{Abstract}

25 Symbiotic interactions change with environmental context. We investigated

26 context-dependence and bet-hedging in the symbiosis between social amoeba hosts and

27 Paraburkholderia bacteria, where the context is the abundance of host food bacteria.

28 Paraburkholderia have been shown to harm hosts dispersed to food-rich environments,

29 but aid hosts dispersed to food-poor environments by allowing hosts to carry food

30 bacteria. Through measuring symbiont density and host spore production, we show that

31 this food context matters in three other ways. First, it matters for symbionts, who suffer a

32 greater cost from competition with food bacteria in the food-rich context. Second, it

33 matters for host-symbiont conflict, changing how symbiont density negatively impacts

34 host spore production. Third, data-based simulations show in some cases this context-

35 dependence can lead to a symbiont-induced bet-hedging advantage for hosts. These

36 results show how food context can have many consequences for the Dictyostelium-

37 Paraburkholderia symbiosis and suggest that symbionts can induce bet-hedging in hosts. 


\section{Introduction}

48 Context-dependence, where the environment can change the sign or magnitude of

49 one partner's effect on the other, is common in symbioses (Bronstein 1994; Thompson

50 1994; Chamberlain et al. 2014). These context-dependent effects on partners can be

51 crucial to understanding the nature of symbiotic interactions (Keeling \& McCutcheon

52 2017; Iwai 2019). For example, in the symbioses between Paramecium bursaria hosts

53 and their Chlorella endosymbionts, hosts benefitted from symbiosis in light

54 environments, but were harmed in the dark. For Chlorella, the effects of symbiosis were

55 negative in co-culture, indicating that hosts exploited their endosymbionts for the benefits

56 hosts receive in light conditions (Lowe et al. 2016). However, in the context of an

57 environment with a Chlorella competitor, hosts benefited their symbionts by eating these

58 competitors (Iwai 2019). This example illustrates that understanding how partners affect

59 each other across multiple contexts can change our view of the interaction, sometimes

60 from one of exploitation to one of mutual benefit.

61 Measures of interaction effects in individual contexts may miss beneficial

62 interactions in symbioses if benefits accrue across contexts at the expense of benefits in

63 any individual context. These benefits that occur across contexts are an example of bet-

64 hedging, where organisms adapt to uncertain environments by avoiding the worst effects

65 of harsh contexts while being suboptimal in more favorable contexts (Slatkin 1974;

66 Philippi \& Seger 1989; Starrfelt \& Kokko 2012). This reduces the variance in fitness

67 across contexts (increasing geometric mean fitness) at the expense of the conventional,

68 arithmetic mean fitness (Seger \& Brockmann 1987). For example, in Lobelia inflata,

69 limiting the timing of reproduction may reduce the variance in fitness from unpredictable 
70 season lengths while being suboptimal in any individual year (Simons \& Johnston 2003).

71 Bet-hedging is thus defined as an adaptation that involves a trade-off between geometric

72 mean fitness and arithmetic mean fitness.

73 Bet-hedging is commonly non-social, but joining together with others may be a

74 powerful way to hedge one's bets against harsh conditions. Cooperative breeding in birds

75 (Rubenstein 2011) and plant-fungus mutualisms (Lekberg \& Koide 2014) have now been

76 suggested as bet-hedging phenotypes. Symbiont-induced bet-hedging may be common

77 because symbionts often give hosts novel traits that allow hosts to gain access to new,

78 sometimes harsh, environments (Moran 2007; Oliver et al. 2010; DiSalvo et al. 2015).

79 For example, endosymbiotic bacteria in aphids play important roles in whether hosts can

80 withstand heat (Montllor et al. 2002) or survive on different host plants (Tsuchida 2004).

81 These novel traits may be more likely to involve bet-hedging if symbionts induce a cost

82 in other contexts so geometric mean fitness is increased at the expense of arithmetic mean

83 fitness. However, symbiont-induced bet-hedging in context-dependent symbioses

84 remains relatively unexplored.

85 Context-dependence is important in the lifecycle of the social amoeba

86 Dictyostelium discoideum. Amoebae need edible bacteria to grow and proliferate (Raper

87 1937), but the abundance (Young 2004; Vos et al. 2013) and quality (Kuserk 1980;

88 Brock et al. 2018) of food bacteria in the soil is known to vary at smaller scales. This

89 results in a patchy environment where some patches are food-rich and other patches are

90 food-poor. In response to starvation, amoebae aggregate and form a multicellular fruiting

91 body to disperse resistant spores to new environments (smith et al. 2014). The patchy soil 
92 environment is considered an important selection pressure for this fruiting body structure

93 (Bonner 1982; Kessin 2001).

94 D. discoideum interacts with three species of mostly inedible Paraburkholderia

95 bacterial symbionts - P. agricolaris, P. hayleyella, and P. bonniae (Brock et al. 2020).

96 Throughout this paper, we will use "Paraburkholderia" or "symbionts" as shorthand for

97 the three symbiotic Paraburkholderia species. Hosts infected with Paraburkholderia

98 have been isolated from multiple locations in the United States with around $25 \%$ of

99 screened hosts being infected by at least one species (Haselkorn et al. 2019).

100 Paraburkholderia are able to enter and live inside $D$. discoideum cells and spores, but can

101 also proliferate, albeit sometimes only slowly, without their hosts (DiSalvo et al. 2015;

102 Shu et al. 2018a; Brock et al. 2020) unlike the obligate endosymbionts that are also found

103 in D. discoideum (Haselkorn et al. 2021). There is some evidence consistent with

104 coevolution between hosts and symbionts (Brock et al. 2016; Shu et al. 2018a; Garcia et

105 al. 2019; Brock et al. 2020). For example, host clones naturally infected with $P$.

106 hayleyella are harmed less by infection with this symbiont than host clones that were not

107 infected in the wild (Shu et al. 2018a) indicating that $P$. hayleyella hosts have adaptations

108 favoring symbiosis. Symbionts also have the ability to move towards hosts (Shu et al.

109 2018b), suggesting that being able to find hosts is beneficial.

110 The symbiosis with Paraburkholderia bacteria impacts the growth and

111 proliferation of $D$. discoideum. Having symbionts allows hosts to carry food bacteria

112 inside the spore-containing part of fruiting bodies called the sorus (DiSalvo et al. 2015).

113 Whether this novel trait is advantageous or not depends on the presence of food bacteria

114 after dispersal. When food is abundant, having symbionts can be costly, as shown by 
115 infected amoebae producing fewer spores than uninfected amoebae (Brock et al. 2011;

116 DiSalvo et al. 2015). In food-poor environments, the cost of having Paraburkholderia is

117 compensated by hosts gaining the ability to carry food bacteria (in addition to inedible

118 Paraburkholderia) in dispersing spores. This allows amoebae to disperse and grow where

119 they ordinarily could not (Brock et al. 2011; DiSalvo et al. 2015). These context-

120 dependent effects on the host could be extremely important in the natural soil

121 environment, where food-poor patches arise frequently (Kessin 2001).

122 Less is known about how symbionts are affected across food contexts. Gaining

123 the ability to disperse to new locations may be a major reason for symbionts to seek out

124 social amoeba hosts (Garcia \& Gerardo 2014), but could also make the context of host

125 food bacteria in the new environment important for symbionts. A benefit from being

126 dispersed to patches with few bacteria could be that symbionts face reduced competition.

127 If few bacteria are present, symbionts will mostly compete with food bacteria that were

128 also carried in the sorus. This should be a relatively low competition situation because

129 symbionts outnumber food bacteria in sori (Khojandi et al. 2019). Having few

130 competitors should advantage symbionts while environments with plentiful bacteria

131 could strongly limit symbiont growth because of their relatively slow growth rates, at

132 least as measured in the lab (Brock et al. 2020). We will use "food-rich" and "food-poor"

133 to describe newly colonized patches with many and few bacteria, respectively, of the sort

134 edible by $D$. discoideum. These categories reflect the relationship to $D$. discoideum and

135 could be called high and low competition in terms of their effect for Paraburkholderia.

136 It is also unclear how the number of extracellular Paraburkholderia in the

137 environment impacts hosts since previous studies have focused on intracellular 
138 Paraburkholderia (Shu et al. 2018b; Miller et al. 2020). When they are outside the

139 amoebae, Paraburkholderia could affect $D$. discoideum fitness through interactions with

140 food bacteria perhaps by reducing the amount of food for hosts through competition or by

141 releasing diffusible toxins that affect amoebae. Thus host food context could also affect

142 the relationship between symbiont density and host spore production.

143 It is also not known whether symbionts can induce a bet-hedging phenotype in

144 hosts. This phenotype would give hosts the ability to evade the harsh effects of food-poor

145 contexts at the expense of reduced fitness in food-rich contexts. This could increase host

146 success across contexts (measured by geometric mean fitness) by reducing the variance in

147 fitness across contexts, but will appear maladaptive according to measures of arithmetic

148 mean fitness (Seger \& Brockmann 1987; Starrfelt \& Kokko 2012). Infected hosts could

149 alternatively gain an outright advantage from carrying symbionts without a trade-off

150 between arithmetic and geometric mean fitness.

151 To understand context-dependence in the symbiosis between amoebae and

152 Paraburkholderia, we used D. discoideum infected with either P. agricolaris or $P$.

153 hayleyella - the two most common and best-studied species of $D$. discoideum

154 symbionts. We investigate whether Paraburkholderia benefit from reduced interspecific

155 competition when dispersed to food-poor contexts, how symbiont density and food

156 context impact host spore production, and whether symbionts induce a bet-hedging

157 phenotype in their hosts when food conditions vary across soil patches.

\section{Methods}


162 uninfected D. discoideum clones, 4 clones naturally infected with $P$. agricolaris, and 4

163 clones naturally infected with P. hayleyella (Figure 1). We cured the infected clones and

164 re-infected them with their native symbionts to equalize infection density. Uninfected

165 clones were left uninfected, but were otherwise treated the same as infected clones. This

166 resulted in three host infection conditions: uninfected, infected with $P$. agricolaris, and

167 infected with P. hayleyella. To mimic natural dispersal, we collected sori and transferred

168 them to food-rich (with additional K. pneumoniae bacteria) or food-poor (KK2 buffer

169 with no K. pneumoniae) nutrient plates. Bacteria appear on food-poor plates only if

170 transferred sori contain bacteria, as expected for infected samples. We grew replicate

171 experimental sets involving all conditions beginning on two separate dates, July 13 and

17222,2020 , and followed up with additional experiments (see results) beginning on January

17326 and April 23, 2021.

175 Paraburkholderia isolation

176 To isolate Paraburkholderia from their hosts, we grew wild collected $D$.

177 discoideum clones on SM/5 plates (2 g glucose (Fisher Scientific), 2 g Bacto Peptone

178 (Oxoid), 2 g yeast extract (Oxoid), $0.2 \mathrm{~g} \mathrm{MgSO}_{4} * 7 \mathrm{H}_{2} \mathrm{O}$ (Fisher Scientific), $1.9 \mathrm{~g}$

$179 \mathrm{KH}_{2} \mathrm{PO}_{4}$ (Sigma-Aldrich), $1 \mathrm{~g} \mathrm{~K}_{2} \mathrm{HPO}_{4}$ (Fisher Scientific), and $15 \mathrm{~g}$ agar (Fisher

180 Scientific) per liter). Wild $D$. discoideum clones were grown with $K$. pneumoniae food

181 bacteria that were suspended in KK2 buffer (2.25 $\mathrm{g} \mathrm{KH}_{2} \mathrm{PO}_{4}$ (Sigma-Aldrich) and $0.67 \mathrm{~g}$

$182 \mathrm{~K}_{2} \mathrm{HPO}_{4}$ (Fisher Scientific) per liter). After wild clones completed the social cycle

183 (feeding, starvation, and fruiting body formation), we collected sori with pipette tips and 
184 placed them on SM/5 plates. We allowed the bacteria and amoebae contained within to

185 proliferate and then streaked out the resulting bacteria to get single colonies.

187 Paraburkholderia removal

188 To generate uninfected clones, we treated infected $D$. discoideum clones with

189 antibiotics by plating on $30 \mu \mathrm{g} / \mathrm{mL}$ tetracycline $\mathrm{SM} / 5$ plates with $200 \mu \mathrm{L}$ of 1.5 optical

190 density $\left(\mathrm{OD}_{600}\right)$ tetracycline-resistant $K$. pneumoniae suspended in KK2 buffer. After

191 passage on $\mathrm{SM} / 5$ plates without tetracycline to let the amoebae recover from any effects

192 of the antibiotic, we collected single sori with a pipette tip and placed ten of them in

193 different locations on $\mathrm{SM} / 5$ plates to confirm that we had successfully removed the

194 bacteria. If bacteria are present, these spot tests will show bacterial growth and

195 Dictyostelium proliferation as the spores hatch and eat the bacteria (Brock et al. 2011).

196 Without bacteria, amoebae cannot proliferate and the spot will stay blank. We considered

197 a clone to be cured if no bacteria showed up in spot tests. We similarly treated naturally

198 uninfected hosts with tetracycline to control for any effect of curing on our results.

200 Paraburkholderia re-infection

201 We re-infected cured D. discoideum clones with their native Paraburkholderia

202 isolates by plating $200 \mu \mathrm{L} 2 \times 10^{5}$ spores with $200 \mu \mathrm{L}$ of $0.1 \%$ Paraburkholderia solution.

203 This solution consisted of $1.5 \mathrm{OD}_{600}$ Paraburkholderia and $1.5 \mathrm{OD}_{600}$ K. pneumoniae in a

204 1:1000 ratio. To confirm re-infection (and also successful isolation), we performed spot

205 tests as above, where successful re-infection was inferred when bacteria grew on 8 or

206 more spots out of the 10 we put down. 
208 Artificial dispersal to food-rich and food-poor plates

209 To obtain sori to transfer to food-rich and food-poor plates, we started by growing

210 D. discoideum clones from frozen stock, as described above, on $200 \mu \mathrm{L} 1.5$ OD $K$.

211 pneumoniae expressing green fluorescent protein (GFP). We obtained GFP-expressing $K$.

212 pneumoniae (strain ID DBS0349837) from the Dicty Stock Center at dictyBase (Fey et

213 al. 2013). This initial growth period is to remove freezer effects and ensure that food

214 bacteria that are carried to new plates are GFP-expressing since stocks were fed non-GFP

215 bacteria before freezing. After six days of growth, we used pipette tips to collect sori

216 from mature fruiting bodies. We counted spores using a hemocytometer and diluted

217 spores to a concentration of $2 \times 10^{5}$ per $\mathrm{mL}$, and then plated them on plates with (food-

218 rich) or without (food-poor) an additional $200 \mu \mathrm{L}$ of the GFP-expressing food bacterium

219 K. pneumoniae (Figure 1). In order to survive on food-poor plates, the host must carry

220 food bacteria from the previous plate. We grew food-rich and food-poor plates for six

221 days unless otherwise stated, enough time for mature fruiting bodies to form.

223 Measurement of bacteria density

224 To measure Paraburkholderia density, we measured the quantity of bacteria left

225 on plates after D. discoideum formed fruiting bodies. We first collected plate contents by

226 washing plates with $15 \mathrm{~mL}$ of KK2 buffer. To remove fruiting bodies and bacteria

227 associated with fruiting bodies, we centrifuged wash solutions for three minutes at 13000

$228 \mathrm{rpm}$. We measured bacteria using optical density measured at $600 \mathrm{~nm}\left(\mathrm{OD}_{600}\right)$, a 
229 frequency at which bacteria commonly scatter light. Because the $\mathrm{OD}_{600}$ is due to both

230 Paraburkholderia and K. pneumoniae, we used GFP fluorescence measurements (with an

231 excitation wavelength of 485 and emission wavelength of $515 \mathrm{~nm}$ ) and a standard curve

232 relating $K$. pneumoniae fluorescence to its $\mathrm{OD}_{600}$ to subtract out the component due to

233 GFP-expressing K. pneumoniae. Both $\mathrm{OD}_{600}$ and fluorescence measures were performed

234 in a 96 well plate with a Tecan Infinite 200 Pro microplate reader.

235 To validate our standard curve, we compared predicted $\mathrm{OD}_{600}$ of P. agricolaris

236 and $K$. pneumoniae to colony forming unit (CFU) counts from the same samples. Linear

237 regression revealed that predicted $\mathrm{OD}_{600}$ measurements explained most of the variation in

238 CFUs, showing that our assay is reliable (Figure S1). We also checked our standard curve

239 for significant quadratic terms, which can cause measurement errors when combining

$240 \mathrm{OD}_{600}$ and fluorescence measures at high densities (Meyers et al. 2018), but our curve did

241 not have a significant quadratic term.

244 To measure host spore production, we estimated spore concentration in the

245 supernatants from washed plates using a hemocytometer. We then calculated the total

246 number of spores per plate by multiplying by the volume of wash solution.

248 Spore production simulations

249 To test whether Paraburkholderia symbionts induce a bet-hedging phenotype in

250 hosts, we simulated repeated bouts of growth and dispersal across soil patches with

251 different probabilities of having food bacteria. Bet hedging occurs when the best 
252 phenotype in terms of arithmetic mean fitness is not the same as the best phenotype in

253 terms of geometric mean fitness (Seger \& Brockmann 1987). We separately modeled

254 three host phenotypes: (1) unifected (2) infected with P. agricolaris, or (3) infected with

255 P. hayleyella. Infections with both species at once are possible, but are rare in nature

256 (Haselkorn et al. 2019) so we exclude coinfections from our analysis.

257 We assumed that environments consisted of $x$ discrete soil patches. In each round

258 of the simulation, patches are randomly assigned as food-rich with probability $p$ or food-

259 poor with probabity 1-p. Thus, in simulations with one patch, populations experience

260 variation in food abundance that is completely temporal. With additional patches,

261 variation can also occur across space. This will reduce temporal variation because fitness

262 at a given time will be summed over multiple patches.

263 Initially all patches are colonized. Each patch produces a number of spores,

264 drawn from the distribution of our empirical spore production values, according to

265 whether it is a food-rich or food-poor patch. Infected hosts have been found to have

266 reduced spore production in food-rich environments that depends on infection density of

267 Paraburkholderia endosymbionts (Brock et al. 2011; DiSalvo et al. 2015; Miller et al.

268 2020). We did not detect this cost in our data, perhaps because we infected with fewer

269 Paraburkholderia. Since these costs appear to vary and could be an important factor

270 affecting fitness in the soil environment when food abundance varies, we included them

271 as a variable. To model costs, we penalized host spore production in food rich

272 environments by multiplying spore production values by $1-c$, where $c$ is the proportion

273 of spores lost because of the cost of infection. When $c$ is 0 , we model the scenario

274 observed in this study, with no infection cost. These cost-adjusted spore production 
275 values are summed to get the total spore production across all patches. This is divided by

$2762 \times 10^{5}$, a rough estimate of the number of spores in a typical sorus, to get the total number

277 of sori, which we are assuming to be the dispersal unit.

278 The global pool of sori is used to seed the next round. Patches are assumed to be

279 empty and are randomly reassigned as food-poor or food-rich. Each sorus is randomly

280 assigned to a patch and it successfully germinates with probability $g$ (we assume $g=0.5$

281 in the main text; other values (0.1 and 0.9) result in similar outcomes (Figure S2\&S3).

282 We assumed that patches colonized by multiple sori were the same as singly colonized

283 patches for the purposes of determining their subsequent spore production. Some patches

284 may remain unfilled.

285 We let cost of infection $c$ and the frequency of food-poor patches $p$ vary from 0 to

2861 in increments of 0.1 and simulated environments with one, 10, and 100 soil patches.

287 We simulated dispersal to new patches for 100 rounds of growth and dispersal using 500

288 replicates for each combination of probability of $p$ and $c$ for each phenotype. At the end

289 of the 100 rounds, we calculated the total spore production per round and calculated

290 geometric mean spore production from these values. To calculate arithmetic mean spore

291 production, we sampled our spore production values from food-rich and food-poor plates

292 according to the number of patches and the value of $p$. We used t tests to compare

293 uninfected hosts' spore production to that of either P. hayleyella or P. agricolaris

294 infected hosts for a given parameter combination. If there was no significant difference

295 between infected and uninfected, we categorized results as "no best phenotype". Because

296 we used many parameter combinations, we corrected p-values for multiple comparisons 
297 using false discovery rate at the level of different numbers of patches and different

298 infection statuses (at the level of individual grids in Figure 4).

\section{$300 \quad$ Statistical Methods}

301 We performed statistics in $\mathrm{R}$ version 3.6.3 (R Core Team 2020). To compare

302 bacteria density and spore production, we used linear mixed models (LMM) with the lme

303 function in the nlme package (Pinheiro \& Bates 2006). To account for random variation

304 from replicate clones and effects of dates when experiments were performed, we included

305 clone and the date the experiment was performed — along with each variable on its own

306 - as random effects. To select the best model of random effects, we used AICc, a

307 sample-size-corrected measure of model fit that balances predictive ability and model

308 complexity (Burnham \& Anderson 2004). Many of our models showed different

309 variances between treatments. To account for these differences in variance, we weighted

310 models with the varIdent function in nlme (Pinheiro \& Bates 2006). We used the

311 emmeans package (Lenth et al. 2018) to perform contrasts.

312 To understand how Paraburkholderia density affects host spore production across

313 food conditions, we fit a LMM using only infected hosts that included symbiont density

314 leftover on plates and whether the plate was food-rich or food-poor, along with the

315 interaction between these variables. We included random effects for clone, date, and both

316 crossed effects and selected the best random effect structure with AICc. We determined

317 whether the interaction was important by comparing AICc of the model including the

318 interaction with models including the other variables but lacking the interaction. 


\section{Results:}

321 Paraburkholderia dispersed by Dictyostelium sori have lower growth when host food

\section{2 bacteria are abundant}

323 The context of a food-poor environment is known to be important for $D$.

324 discoideum hosts. It is not known how Paraburkholderia are affected by this same

325 context, but reduced competition with food bacteria seems likely. We tested this by

326 growing infected sorus contents on food-poor and food-rich nutrient plates and measuring

327 the density of Paraburkholderia after D. discoideum fruiting body formation (Figure 1).

328 After infected hosts formed fruiting bodies, Paraburkholderia densities were lowest in

329 food-rich conditions (Figure 2A), as expected if they compete with food bacteria. There

330 was around five times more $P$. agricolaris on food-poor than food-rich plates (LMM, $<<$

331 0.001). P. hayleyella growth was higher in food-poor conditions than food-rich, but this

332 difference was not significant after 6 days $(\mathrm{LMM}, \mathrm{p}=0.416)$. Because $P$. hayleyella

333 grows slowly, we performed two more experiments with P. hayleyella with 8 and 12-day

334 growth periods (Figure 2B). Allowing for longer incubations did not result in

335 significantly higher density of $P$. hayleyella $(\mathrm{LMM}, \mathrm{p}=0.633)$, suggesting that $P$.

336 hayleyella reach their maximum density at or before 6 days, but including these

337 additional experiments gave us enough power to find a significant increase in $P$.

338 hayleyella density in food-poor conditions relative to food-rich (LMM, $\mathrm{p}=0.027)$. These

339 results show that symbiont density is context-dependent.

341 Higher symbiont density harms hosts, but less so in food-poor contexts 
The host-food context may affect the relationship between symbiont density and

343 host spore production and therefore the degree of conflict or cooperation between them.

344 To investigate this, we also measured total host spore production from plates where we

345 measured the growth of Paraburkholderia symbionts (Figure 1). We used uninfected

346 hosts as a baseline for fitness without symbionts. We confirmed prior studies (Brock et

347 al. 2011; DiSalvo et al. 2015) showing that infected hosts could carry food bacteria and

348 proliferate on food-poor plates, while uninfected host could not (Figure 3A).

349 Surprisingly, we did not observe a cost of being infected in food-rich conditions $(\mathrm{p}>0.5$

350 for both species) which has been seen in previous studies (Brock et al. 2011; DiSalvo et

351 al. 2015; Shu et al. 2018a). This is likely a result of our lower infection dosage of $0.1 \%$.

352 While having some symbionts is essential for hosts to be able to carry food and

353 survive in food-poor conditions, higher symbiont densities may nevertheless harm hosts,

354 perhaps in ways that depend on food context. We found that larger populations of

355 symbionts as measured by $\mathrm{OD}_{600}$ were associated with lower host spore production, but

356 this harm was reduced in food-poor conditions. Lower host spore production was

357 associated with being in a food-poor environment $\left(\beta_{\text {food-poor }}=-3.283\right.$, se $\left.=0.853\right)$ and

358 symbiont density $\left(\beta_{\text {density }}=-10.317\right.$, se $\left.=6.364\right)$, but the interaction between food scarcity

359 and symbiont density showed that the harmful effect of higher symbiont densities was

360 lessened on food-poor plates $\left(\beta_{\text {food-poor } * \text { density }}=8.078, \mathrm{se}=6.381\right.$; Figure $\left.3 \mathrm{~B}\right)$. These

361 results indicate that symbiont density may come at the expense of host spore production,

362 but that this cost decreases in food-poor environments.

364 Symbionts can induce a bet-hedging phenotype in hosts 
365 Because Paraburkholderia helps hosts in food-poor contexts, we hypothesized

366 that they could give hosts a bet-hedging advantage that increases geometric mean fitness

367 at the expense of arithmetic mean fitness across spatial and temporal variation in food

368 abundance in the soil. We modeled this by using our empirical spore production values to

369 simulate 100 rounds of growth and dispersal across environments with one, 10, and 100

370 soil patches. We modeled three symbiosis phenotypes: (1) uninfected, (2) infected with

371 P. agricolaris, and (3) infected with P. hayleyella (Figure 4A, Animations S1-3) in their

372 own separate environments and then compared their geometric and arithmetic mean spore

373 productions.

374 Soil patches were either food-rich or food-poor and changed randomly in each

375 round. By changing the number of patches, we are able to understand how host fitness is

376 affected when variation in food abundance is more temporal (few soil patches) or spatial

377 (many soil patches). We varied the frequency of food-poor soil patches and calculated a

378 phenotype's success as the geometric or arithmetic mean spore production. We included

379 the cost of infection observed in other studies (DiSalvo et al. 2015; Shu et al. 2018a;

380 Haselkorn et al. 2019; Miller et al. 2020) by subtracting some proportion of spores from

381 our empirical measures for infected hosts in food-rich conditions. We classified a

382 phenotype as a bet-hedger if the best phenotype in terms of geometric mean fitness

383 differed from the best phenotype in terms of arithmetic mean fitness (Figure 4B).

384 We found that uninfected hosts were favored by both geometric and arithmetic

385 means when costs of infection were high, food was abundant, and food abundance

386 variation was spatial (Figure 4C\&D; pink). Associating with Paraburkholderia,

387 especially $P$. hayleyella, provided an advantage in terms of arithmetic mean fitness across 
388 a wide range of parameters, even with fairly large costs (Figure 4C\&D; blue). Symbionts

389 were most beneficial when variation in food abundance was temporal (one soil patch). As

390 the number of patches in the environment increased, the benefits of having symbionts

391 decreased, with $P$. agricolaris being beneficial only when the probability of food was

392 high. This lowered benefit of $P$. agricolaris is especially apparent when fewer potential

393 dispersers manage to colonize new patches (Figure S2).

394 We found that bet-hedging is likely when variation in food abundance is more

395 temporal (one patch), when food-poor patches are common, and when costs of infection

396 are high (Figure 4C\&D; green). This bet-hedging advantage in more temporally varying

397 environments is because uninfected populations go extinct when generations without

398 food occur (as illustrated in Figure 4A with a single soil patch, Animation S1). Bet-

399 hedging becomes rarer as the number of patches increases (middle and rightmost panels

400 in Figure 4C\&D) and the worst consequences of food-poor patches (extinction) become

401 rare due to the presence of some patches with food. Bet-hedging occurs across a broader

402 range of parameters in P. agricolaris simulations (Figure 4C). These results show that

403 symbionts can provide an outright benefit for hosts in many conditions and can induce a

404 bet-hedging phenotype in some conditions.

405

406 Discussion

407 Our results show how the context of host food abundance affects the

408 Dictyostelium-Paraburkholderia symbiosis beyond the previously demonstrated

409 advantage to hosts when food is rare (Brock et al. 2011). We found evidence that both

410 Paraburkholderia species benefit from reduced competition when they are carried to 
411 food-poor environments (Figure 2). Symbiont density negatively affected host spore

412 production, but symbionts harmed hosts less in food-poor conditions (Figure 3B).

413 Symbionts gave hosts an outright or a bet-hedging advantage across a range of

414 parameters in patchy soils (Figure 4).

415 Our finding that symbionts benefit from reduced competition when dispersed to

416 food-poor environments fits in with other findings of competitive benefits for symbionts

417 (Iwai 2019). Competition between symbionts and food bacteria may also be the cause of

418 context-dependent effects of symbiont density on host spore production. Our spore

419 production results showed that higher symbiont densities resulted in lower host spore

420 production, indicating that symbionts are harmful to hosts. However, higher symbiont

421 densities are less harmful in food-poor conditions when competition is lower (Figure 3B).

422 The reduced harm for hosts could be the result of less antagonism between bacteria,

423 which results in less collateral damage to amoebae through secreted toxins or other

424 competitive interactions between food bacteria and symbionts. The generality of our

425 results is limited somewhat by only using one species of food bacteria. While using a

426 single food bacterium is more experimentally tractable, amoebae encounter multiple

427 bacteria species in their natural environments (Brock et al. 2018). Different species, or

428 combinations of species, could change competition with symbionts and affect host spore

429 production in different ways.

430 Paraburkholderia symbionts benefit hosts by giving hosts the ability to carry food

431 to food-poor contexts (Brock et al. 2011; DiSalvo et al. 2015). Using simulations, we

432 found that this benefit was prevalent across a range of costs, food probabilities, and

433 number of soil patches according to measures of arithmetic mean spore production 
434 (Figure 4). Hosts could also benefit across contexts through bet-hedging, where

435 geometric mean fitness trades off with arithmetic mean fitness (Seger \& Brockmann

436 1987). Bet-hedging occurred more often in our simulations when costs imposed by the

437 symbiont were relatively high and food abundance varied temporally (few soil patches).

438 Temporal variation disadvantaged uninfected amoebae because they went extinct in food-

439 poor patches while infected amoebae weathered these harsh contexts. These results are

440 interesting when paired with the finding that infected hosts constitute about $25 \%$ of the

441 population (Haselkorn et al. 2019). This may mean that symbiosis is not universally

442 favored - if so, Paraburkholderia infection would be fixed in the population — but

443 instead depends on ecological conditions.

444 Our simulations were necessarily simplified to make them tractable. Two notable

445 limitations from this are that soil patches are discrete and equally sized and that host

446 spore production as measured in the lab may not accurately reflect nature. Though not

447 unique to our model (Starrfelt \& Kokko 2012), our finding that bet hedging is most likely

448 in temporally varying environments also limits the scope of our results. However, such

449 temporal variation may result naturally in the soil from rainfall variation and has been

450 shown to impact soil bacteria abundance (Barnard et al. 2013). Though it remains

451 uncertain how much Paraburkholderia induces host bet-hedging in the wild, our model

452 illustrates the potential for a symbiotic partner to induce bet-hedging.

453 Our simulation results suggest that bet-hedging may be an important, but

454 underappreciated, consequence of some symbioses. Bet-hedging has been suggested in

455 some symbioses (Lekberg \& Koide 2014), but has not been tested in detail. Though our

456 simulations were tailored to the symbiosis between D. discoideum and Paraburkholderia, 
457 they should apply to other cases of context-dependent symbiosis with costs and temporal

458 variation. Since context-dependence is common in symbioses (Bronstein 1994), we

459 expect that bet-hedging that involves symbiosis may be relatively common. Accounting

460 for geometric mean fitness in symbiotic interactions will be crucial to determining the

461 true extent of bet-hedging in symbioses.

462 Bet-hedging may be especially relevant in symbioses where hosts gain novel

463 phenotypes from symbionts (Moran 2007; Oliver et al. 2010) because they can allow

464 symbionts to survive in new, previously harsh, contexts (Montllor et al. 2002; Tsuchida

465 2004). However, it is important to note that bet-hedging will not evolve unless these

466 harsh contexts occur probabilistically. If the new harsh environment is constant,

467 symbiosis will be favored without any bet-hedging. If the harsh environment is too

468 predictable, plasticity will be favored instead of bet-hedging (Botero et al. 2015). Thus an

469 understanding of the ecological contexts will also be important for identifying symbiont-

470 induced bet-hedging.

472 Acknowledgements: This material is based upon work funded by the National Science

473 Foundation under grants DEB-1753743 and IOS-1656756. We thank Tyler Larsen and

474 James Medina for comments on the manuscript. We would also like to thank the

475 Strassmann/Queller lab group, especially Debbie Brock, for feedback during the

476 development and execution of this project.

477 Authorship statement: TS, DQ, and JS designed the study and wrote the manuscript. TS

478 performed the experiments and simulations and analyzed the data. 
481 Data Statement: Data will be archived at Dryad upon acceptance. Data and code for

482 simulations and analysis will be available at www.gitlab.com/treyjscott/farmerBH.

\section{References}

485 Barnard, R.L., Osborne, C.A. \& Firestone, M.K. (2013). Responses of soil bacterial and fungal communities to extreme desiccation and rewetting. ISME J., 7, 22292241.

Bonner, J.T. (1982). Evolutionary Strategies and Developmental Constraints in the Cellular Slime Molds. Am. Nat., 119, 530-552.

Botero, C.A., Weissing, F.J., Wright, J. \& Rubenstein, D.R. (2015). Evolutionary tipping points in the capacity to adapt to environmental change. Proc. Natl. Acad. Sci., 112, 184-189.

Brock, D.A., Douglas, T.E., Queller, D.C. \& Strassmann, J.E. (2011). Primitive agriculture in a social amoeba. Nature, 469, 393-396.

Brock, D.A., Haselkorn, T.S., Garcia, J.R., Bashir, U., Douglas, T.E., Galloway, J., et al. (2018). Diversity of Free-Living Environmental Bacteria and Their Interactions With a Bactivorous Amoeba. Front. Cell. Infect. Microbiol., 8.

Brock, D.A., Jones, K., Queller, D.C. \& Strassmann, J.E. (2016). Which phenotypic traits of Dictyostelium discoideum farmers are conferred by their bacterial symbionts? Symbiosis, 68, 39-48.

Brock, D.A., Noh, S., Hubert, A.N.M., Haselkorn, T.S., DiSalvo, S., Suess, M.K., et al. (2020). Endosymbiotic adaptations in three new bacterial species associated with Dictyostelium discoideum : Paraburkholderia agricolaris sp. nov., Paraburkholderia hayleyella sp. nov., and Paraburkholderia bonniea sp. nov. PeerJ, 8, e9151.

Bronstein, J.L. (1994). Conditional outcomes in mutualistic interactions. Trends Ecol. Evol., 9, 214-217.

Burnham, K.P. \& Anderson, D.R. (2004). Multimodel Inference: Understanding AIC and BIC in Model Selection. Sociol. Methods Res., 33, 261-304.

Chamberlain, S.A., Bronstein, J.L. \& Rudgers, J.A. (2014). How context dependent are species interactions? Ecol. Lett., 17, 881-890.

DiSalvo, S., Haselkorn, T.S., Bashir, U., Jimenez, D., Brock, D.A., Queller, D.C., et al. (2015). Burkholderia bacteria infectiously induce the proto-farming symbiosis of Dictyostelium amoebae and food bacteria. Proc. Natl. Acad. Sci., 112, E5029-E5037.

Fey, P., Dodson, R.J., Basu, S. \& Chisholm, R.L. (2013). One stop shop for everything Dictyostelium: dictyBase and the Dicty Stock Center in 2012. In: Dictyostelium Discoideum Protocols. Springer, pp. 59-92.

Garcia, J.R. \& Gerardo, N.M. (2014). The symbiont side of symbiosis: do microbes really benefit? Front. Microbiol., 5. 
521 Garcia, J.R., Larsen, T.J., Queller, D.C. \& Strassmann, J.E. (2019). Fitness costs and

522

523

524

525

526

527

528

529

530

531

532

533

534

535

536

537

538

539

540

541

542

543

544

545

546

547

548

549

550

551

552

553

554

555

556

557

558

559

560

561

562

563

564

565 benefits vary for two facultative Burkholderia symbionts of the social amoeba, Dictyostelium discoideum. Ecol. Evol., ece3.5529.

Haselkorn, T.S., DiSalvo, S., Miller, J.W., Bashir, U., Brock, D.A., Queller, D.C., et al. (2019). The specificity of Burkholderia symbionts in the social amoeba farming symbiosis: Prevalence, species, genetic and phenotypic diversity. Mol. Ecol., 28, 847-862.

Haselkorn, T.S., Jimenez, D., Bashir, U., Sallinger, E., Queller, D.C., Strassmann, J.E., et al. (2021). Novel Chlamydiae and Amoebophilus endosymbionts are prevalent in wild isolates of the model social amoeba Dictyostelium discoideum. Environ. Microbiol. Rep., 1758-2229.12985.

Iwai, S. (2019). Photosynthetic Endosymbionts Benefit from Host's Phagotrophy, Including Predation on Potential Competitors. Curr. Biol., 29, 3114-3119.

Keeling, P.J. \& McCutcheon, J.P. (2017). Endosymbiosis: The feeling is not mutual. J. Theor. Biol., 434, 75-79.

Kessin, R.H. (2001). Dictyostelium: evolution, cell biology, and the development of multicellularity. Cambridge University Press.

Khojandi, N., Haselkorn, T.S., Eschbach, M.N., Naser, R.A. \& DiSalvo, S. (2019). Intracellular Burkholderia Symbionts induce extracellular secondary infections; driving diverse host outcomes that vary by genotype and environment. ISME J.

Kuserk, F.T. (1980). The Relationship Between Cellular Slime Molds and Bacteria in Forest Soil. Ecology, 61, 1474-1485.

Lekberg, Y. \& Koide, R.T. (2014). Integrating physiological, community, and evolutionary perspectives on the arbuscular mycorrhizal symbiosis. Botany, 92, 241-251.

Lenth, R., Singmann, H., Love, J., Buerkner, P. \& Herve, M. (2018). Emmeans: Estimated marginal means, aka least-squares means. $R$ Package Version, 1, 3.

Lowe, C.D., Minter, E.J., Cameron, D.D. \& Brockhurst, M.A. (2016). Shining a Light on Exploitative Host Control in a Photosynthetic Endosymbiosis. Curr. Biol., 26, 207-211.

Meyers, A., Furtmann, C. \& Jose, J. (2018). Direct optical density determination of bacterial cultures in microplates for high-throughput screening applications. Enzyme Microb. Technol., 118, 1-5.

Miller, J.W., Bocke, C.R., Tresslar, A.R., Schniepp, E.M. \& DiSalvo, S. (2020). Paraburkholderia Symbionts Display Variable Infection Patterns That Are Not Predictive of Amoeba Host Outcomes. Genes, 11, 674.

Montllor, C.B., Maxmen, A. \& Purcell, A.H. (2002). Facultative bacterial endosymbionts benefit pea aphids Acyrthosiphon pisum under heat stress. Ecol. Entomol., 27, 189-195.

Moran, N.A. (2007). Symbiosis as an adaptive process and source of phenotypic complexity. Proc. Natl. Acad. Sci., 104, 8627-8633.

Oliver, K.M., Degnan, P.H., Burke, G.R. \& Moran, N.A. (2010). Facultative Symbionts in Aphids and the Horizontal Transfer of Ecologically Important Traits. Annu. Rev. Entomol., 55, 247-266. 
566 Philippi, T. \& Seger, J. (1989). Hedging one’s evolutionary bets, revisited. Trends

$567 \quad$ Ecol. Evol., 4, 41-44.

568 Pinheiro, J. \& Bates, D. (2006). Mixed-effects models in S and S-PLUS. Springer Science

569 \& Business Media.

570 R Core Team. (2020). R: A language and environment for statistical computing. R

$571 \quad$ Foundation for Statistical Computing, Vienna, Austria.

572 Raper, K.B. (1937). Growth and development of Dictyostelium discoideum with

573

574

575

576

577

578

579

580

581

582

583

584

585

586

587

588

589

590

591

592

593 different bacterial associates. J. Agric. Res., 55, 289-316.

Rubenstein, D.R. (2011). Spatiotemporal environmental variation, risk aversion, and the evolution of cooperative breeding as a bet-hedging strategy. Proc. Natl. Acad. Sci., 108, 10816-10822.

Seger, J. \& Brockmann, H. (1987). What is bet-hedging? In: Oxford surveys in evolutionary biology. Oxford University Press.

Shu, L., Brock, D.A., Geist, K.S., Miller, J.W., Queller, D.C., Strassmann, J.E., et al. (2018a). Symbiont location, host fitness, and possible coadaptation in a symbiosis between social amoebae and bacteria. eLife, 7, 25.

Shu, L., Zhang, B., Queller, D.C. \& Strassmann, J.E. (2018b). Burkholderia bacteria use chemotaxis to find social amoeba Dictyostelium discoideum hosts. ISME J., 12, 1977-1993.

Simons, A.M. \& Johnston, M.O. (2003). Suboptimal timing of reproduction in Lobelia inflata may be a conservative bet-hedging strategy. J EVOL BIOL, 11.

Slatkin, M. (1974). Hedging one's evolutionary bets. Nature, 250, 704-705.

smith, jeff, Queller, D.C. \& Strassmann, J.E. (2014). Fruiting bodies of the social amoeba Dictyostelium discoideum increase spore transport by Drosophila. BMC Evol. Biol., 14, 105.

Starrfelt, J. \& Kokko, H. (2012). Bet-hedging-a triple trade-off between means, variances and correlations. Biol. Rev., 87, 742-755.

594

595

596

597

598

599

Thompson, J.N. (1994). The coevolutionary process. University of Chicago Press.

Tsuchida, T. (2004). Host Plant Specialization Governed by Facultative Symbiont. Science, 303, 1989-1989.

Vos, M., Wolf, A.B., Jennings, S.J. \& Kowalchuk, G.A. (2013). Micro-scale determinants of bacterial diversity in soil. FEMS Microbiol. Rev., 37, 936-954.

Young, I.M. (2004). Interactions and Self-Organization in the Soil-Microbe Complex. Science, 304, 1634-1637.

600

601

602

603

604

605 


\section{Figure Legends}

Exferimental Set-up

Food-rich Food-poor

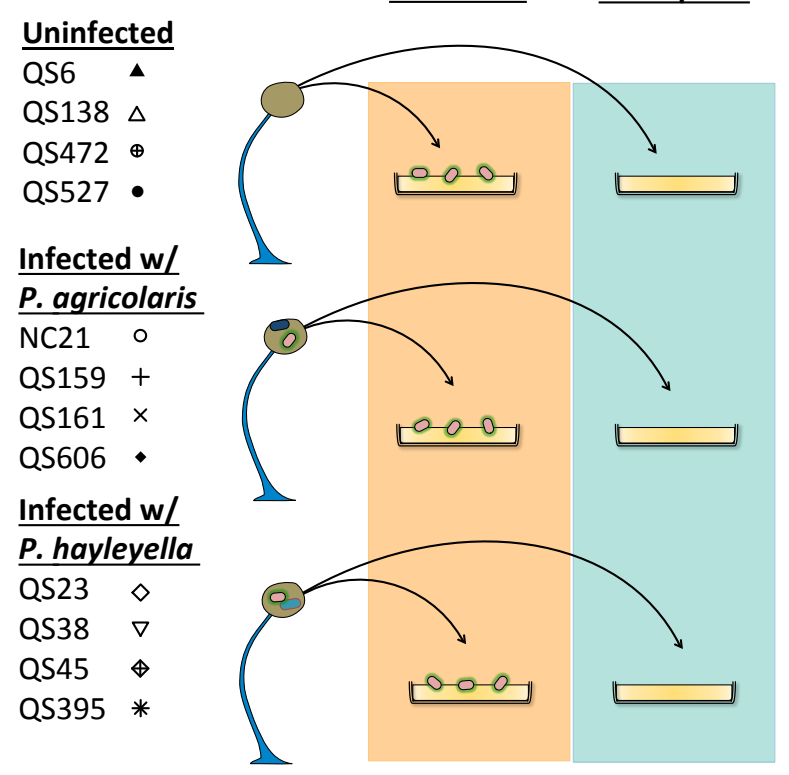

B Fitness Measurement

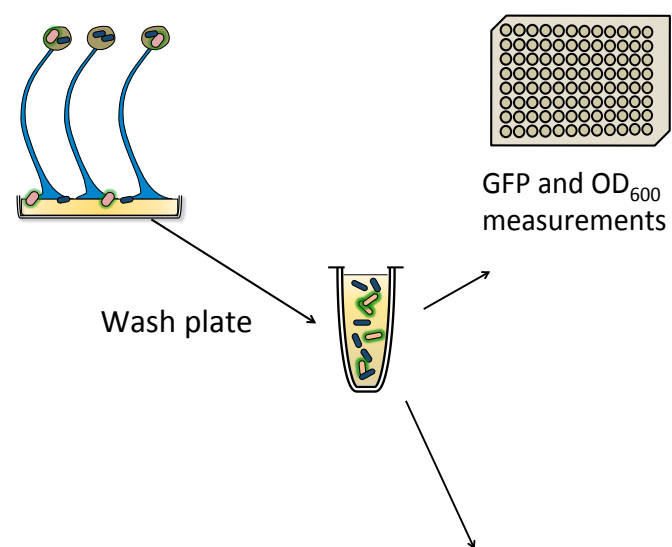

Key

K. pneumoniae (GFP) 0

P. agricolaris

P. hayleyella
Host spore counts

608 Figure 1: Schematic of experimental design. (A) Uninfected and infected D. discoideum

609 fruiting bodies are collected and plated on food-rich and food-poor plates (after one

610 passage on GFP-expressing K. pneumoniae food bacteria). These plates are grown for six

611 days and then washed for bacterial measurement and spore counting (B). Bacteria are

612 measured by calculating GFP fluorescence and optical density (see Methods).

613

614 

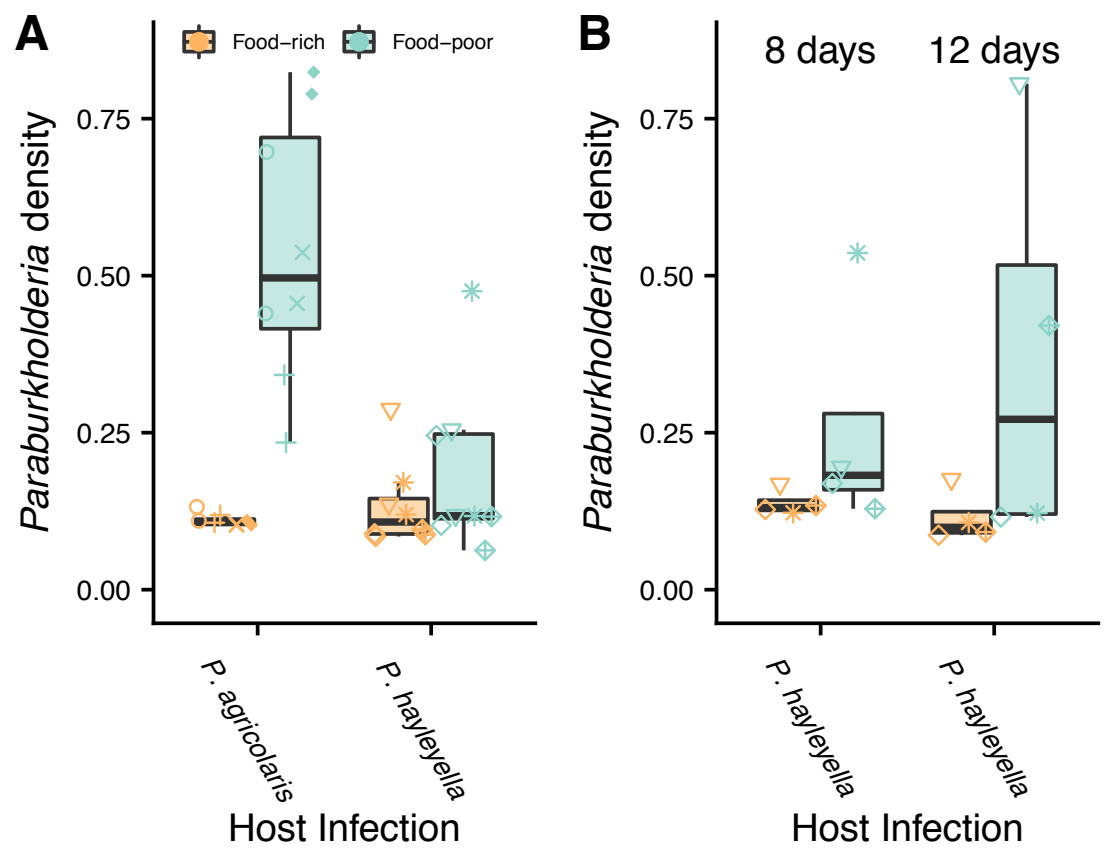

615

616 Figure 2: More Paraburkholderia were recovered from plates after fruiting body

617 formation from food-poor plates (those that had not received additional K. pneumoniae).

618 (A) Paraburkholderia density after 6 days. (B) P. hayleyella density after 8 and 12 days.

619 Point shapes show individual clones 4 of each (see Figure 1).

620

621 

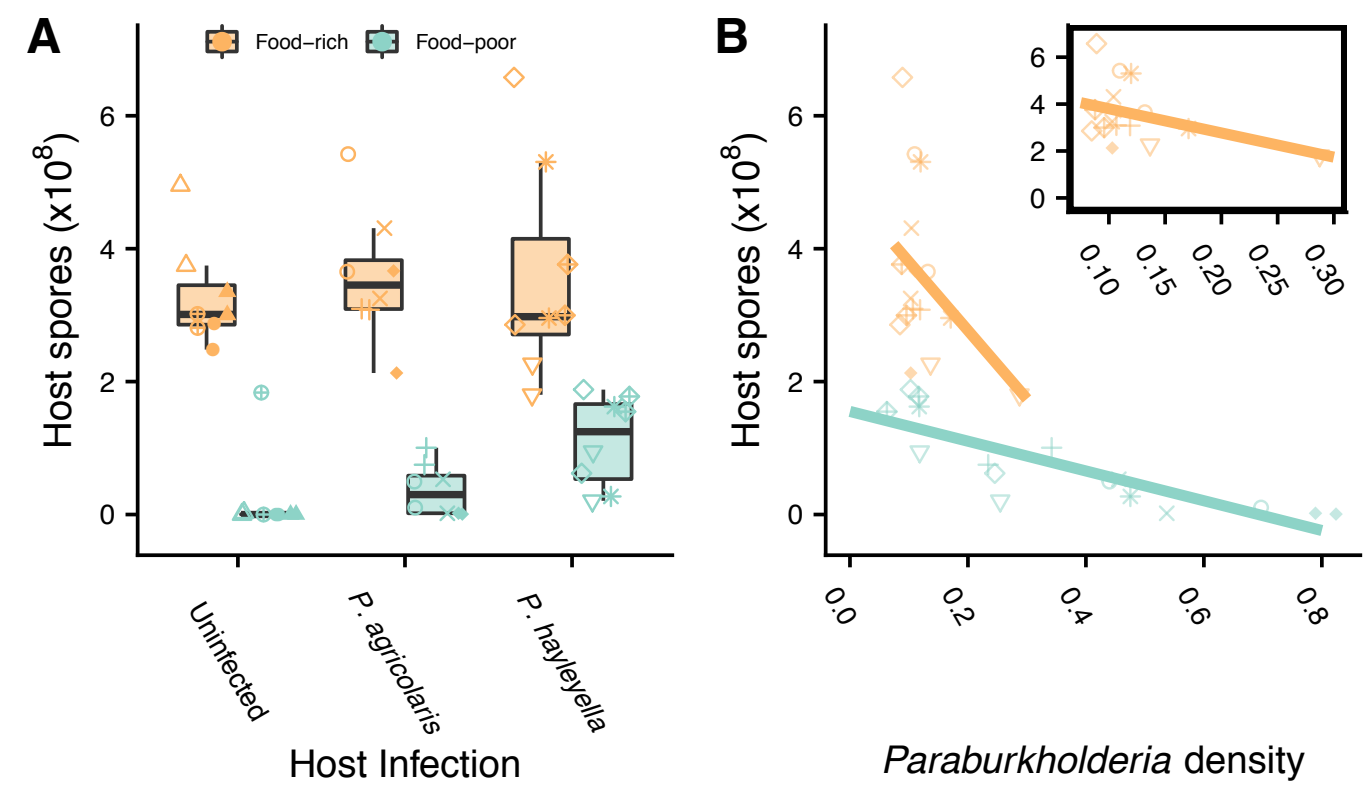

623 Figure 3: Effects of Paraburkholderia infection and density on host spore production.

624 (A) Spore production of hosts from food-rich and food-poor plates for uninfected, $P$.

625 agricolaris infected, and $P$. hayleyella infected hosts. (B) Interaction between measured

626 Paraburkholderia density $\left(\mathrm{OD}_{600}\right)$ and food environment on host spore production. This

627 interaction model explained $95 \%$ of the variance in spore production. Inset shows food-

628 rich results on smaller scale. Point shapes show individual clones (see Figure 1). 

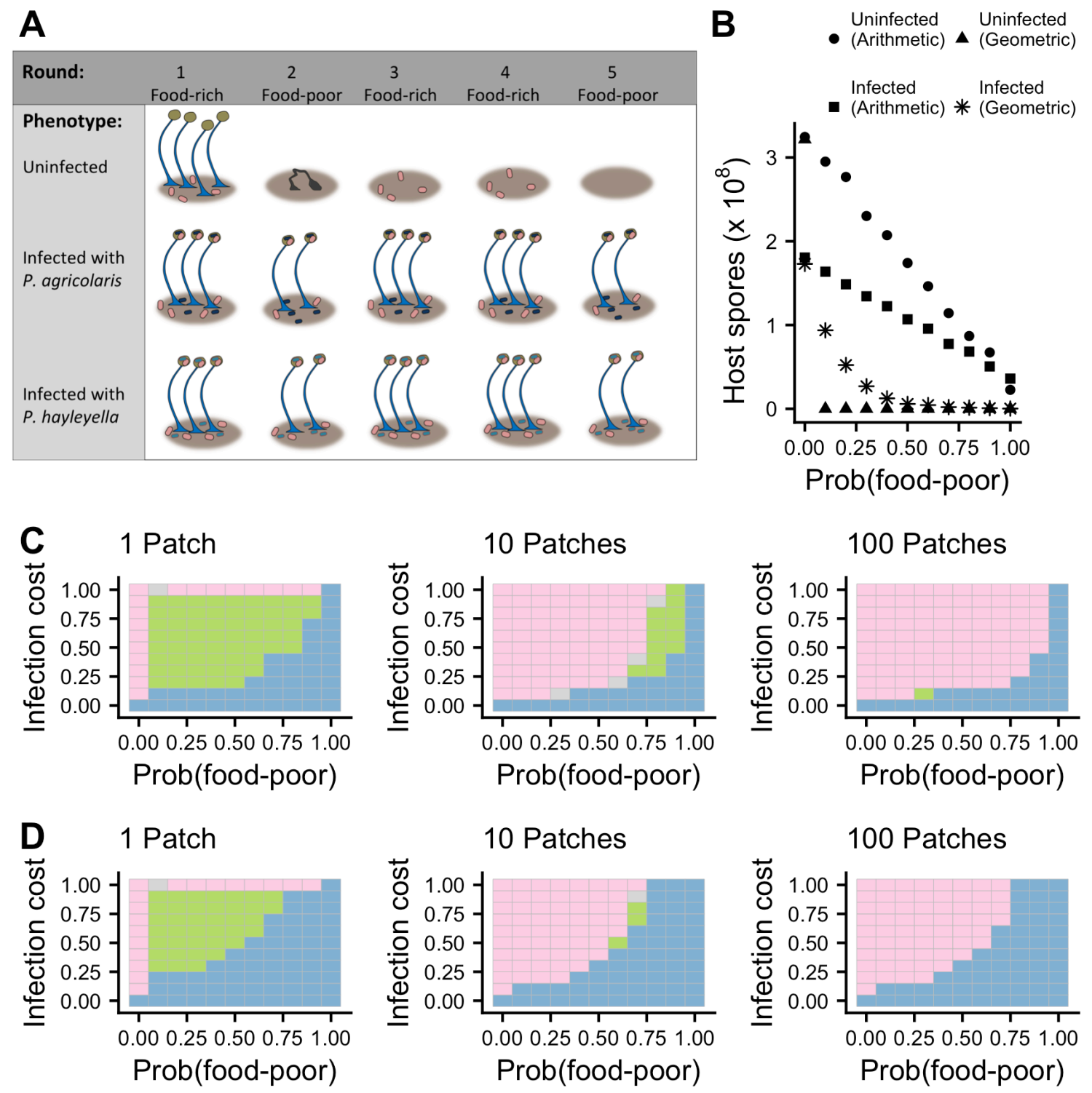

Uninfected Infected

Infected (Bet-hedging)

No Best Phenotype

631 Figure 4: Benefits of associating with Paraburkholderia symbionts depends on variation

632 in food availability, fitness costs, and number of soil patches. (A) Toy example of

633 simulations with five rounds of growth and dispersal where spores are dispersed to a

634 single soil patch each generation. Lack of food could lead to the extinction of uninfected

635 clones. (B) Illustration of bet-hedging trade-off between geometric and arithmetic mean

636 spore production in a single soil patch. Bet-hedging occurs when the food-poor

637 probability is between 0.1 and 0.7 (note how uninfected hosts have the highest arithmetic 
638 mean while infected hosts have the highest geometric mean). Infected hosts suffered a

$63950 \%$ reduction in spore production in these simulations. Performance of $P$. agricolaris

640 (C) and P. hayleyella (D) relative to uninfected for 1, 10, and 100 soil patches. Pink

641 shows when uninfected hosts have higher arithmetic and geometric mean spore

642 production; blue shows when infected hosts have higher arithmetic and geometric mean

643 spore production; green shows when arithmetic fitness is reduced for higher geometric

644 mean fitness (bet-hedging).

645

646

647

648

649

650

651

652

653

654

655

656

657

658

659

660 


\section{Supplemental Material}

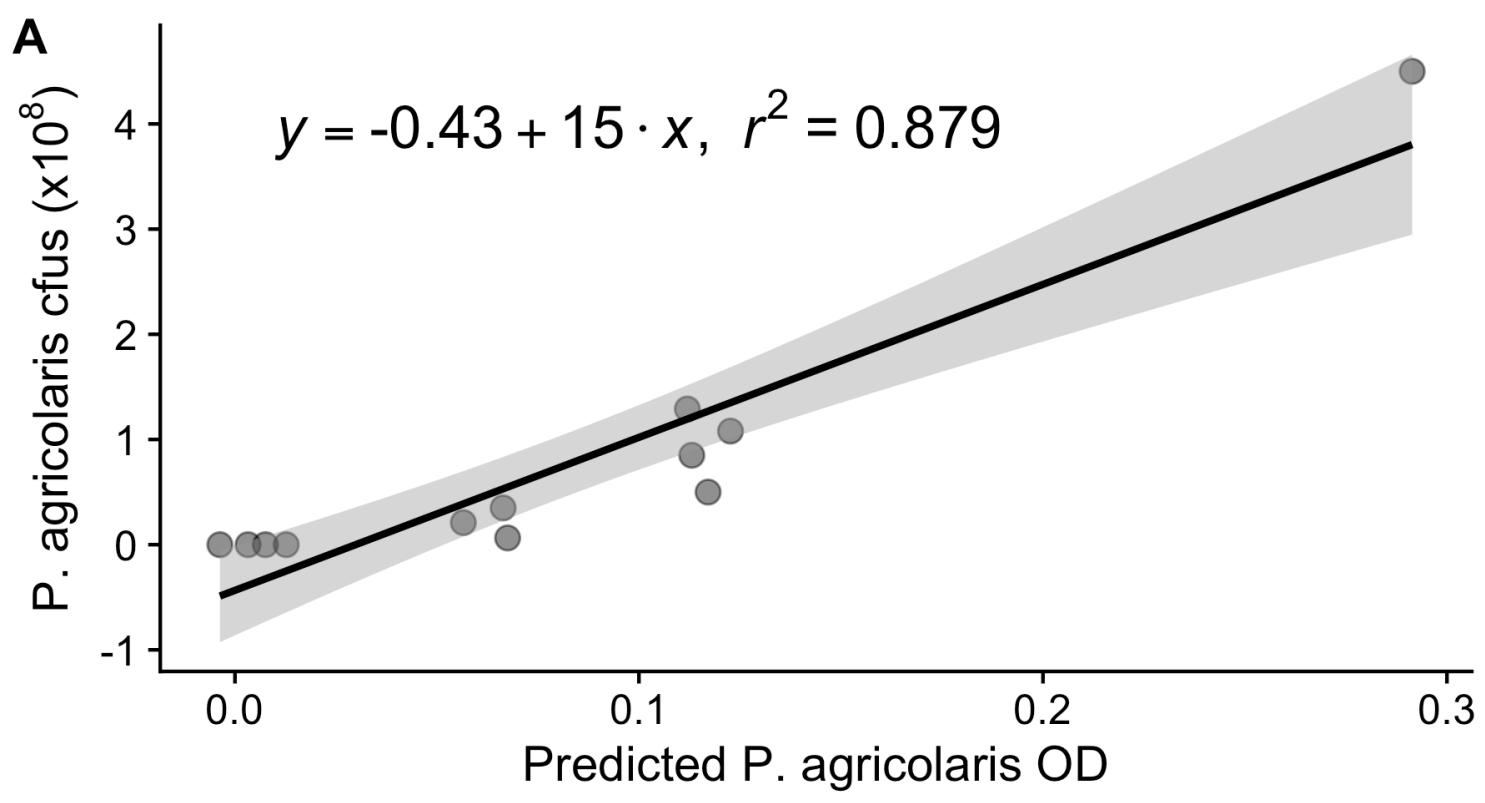

662

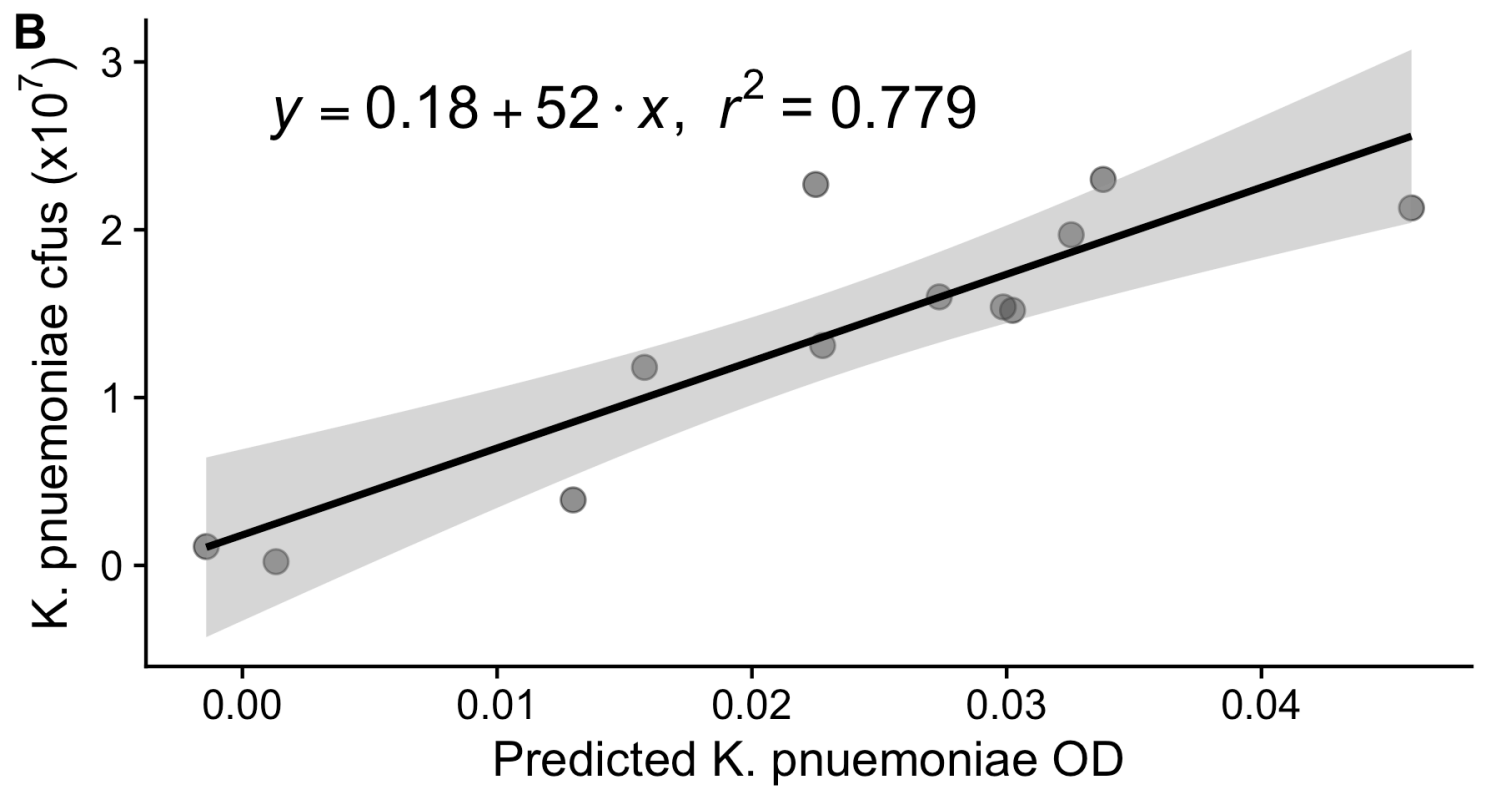

663 Figure S1: Linear regression results relating estimated optical density (predicted from

664 standard curve) to colony counts from serial dilutions of the same P. agricolaris (A) and 665 K. pneumoniae (B) samples. 
A 1 Patch

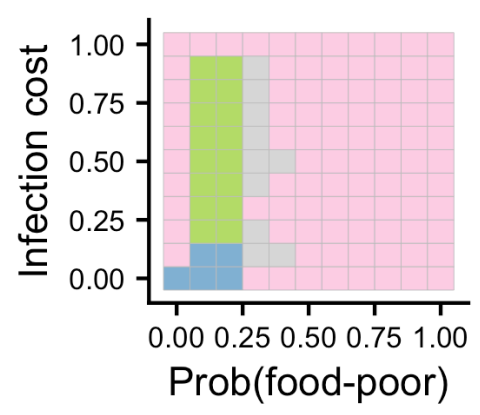

B 1 Patch

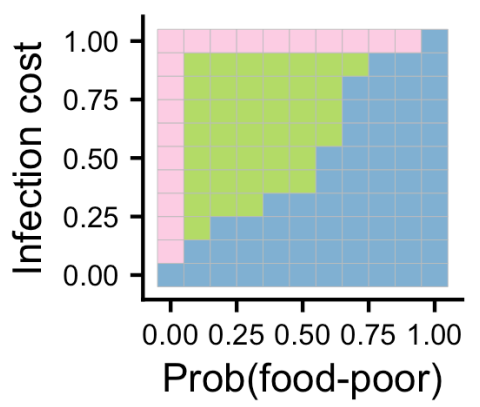

10 Patches

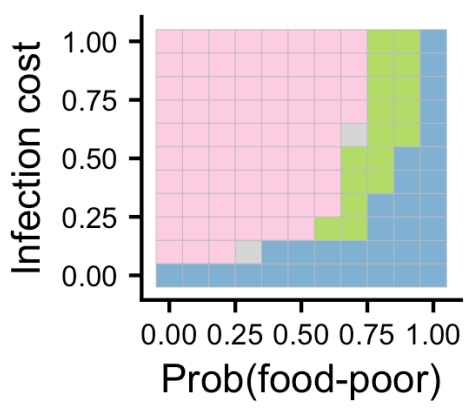

10 Patches

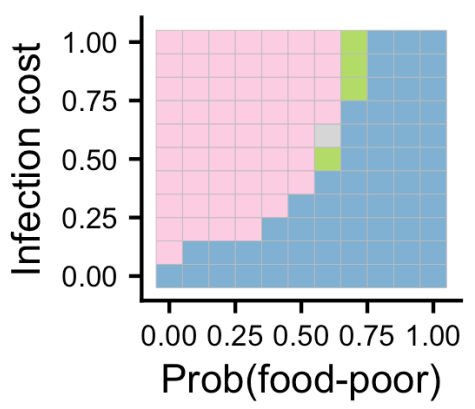

100 Patches

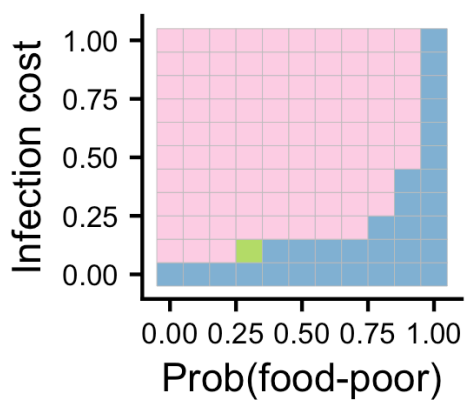

100 Patches

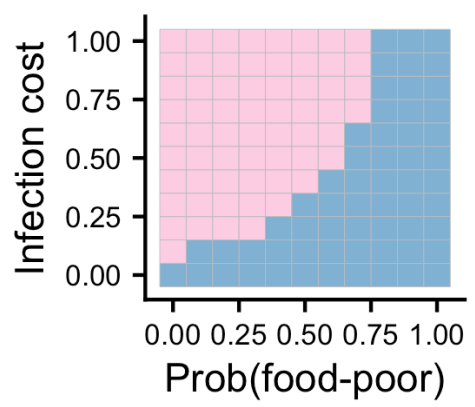

No Best Phenotype

670 Figure S2: Plot of the best phenotype for a given probability of being in a no food

671 environment and cost of having symbionts. These are the same as Figure $4 \mathrm{C}$ and D with $10 \%$ of sori successfully germinating. 


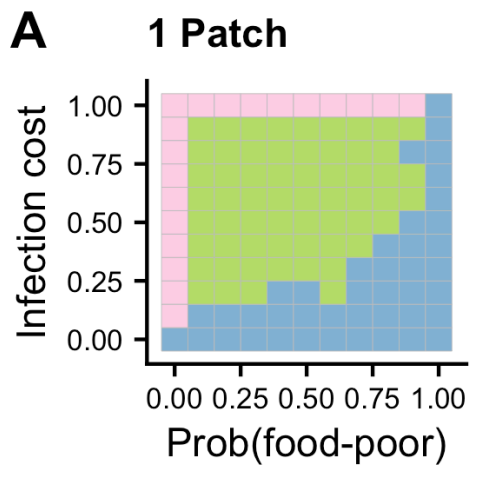

B 1 Patch

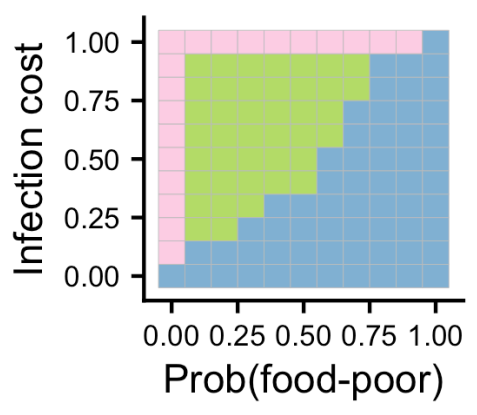

674

675 Figure S3: Plot of the best phenotype for a given probability of being in a no food

676 environment and cost of having symbionts. These are the same as Figure 4C-H with $90 \%$

677 of sori successfully germinating.

678

679 Animation S1: Example simulation with 100 rounds of growth and dispersal with one

680 soil patch, a $50 \%$ cost of infection, and a food-poor frequency of 0.3 .

681

682 Animation S2: Example simulation with 100 rounds of growth and dispersal with 10 soil 683 patches, a $50 \%$ cost of infection, and a food-poor frequency of 0.3 .

684

685 Animation S3: Example simulation with 100 rounds of growth and dispersal with 100

686 soil patches, a 50\% cost of infection, and a food-poor frequency of 0.3 .
100 Patches

100 Patches

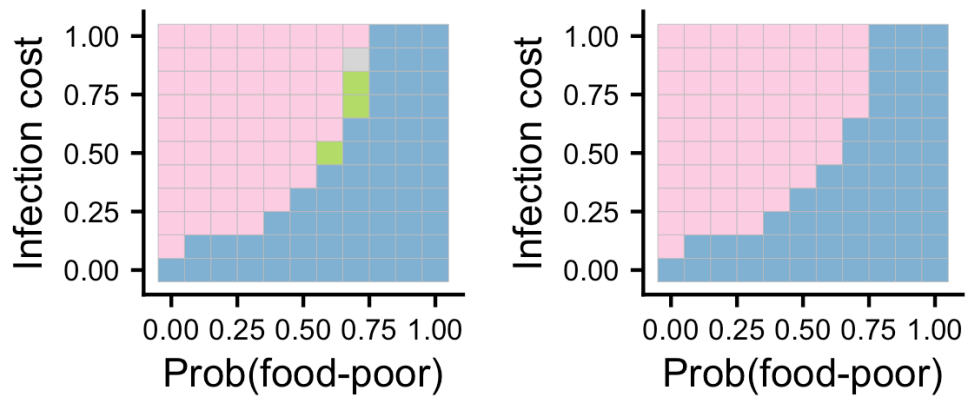

No Best Phenotype 